\title{
PASSIVE BANDWIDTH ESTIMATION ENABLED ROUTING PROTOCOL FOR MOBILE AD HOC NETWORK
}

\author{
Rajeev Kumar \\ Research Scholar \\ Department of CSE \\ JSSATE, Bangalore, Karnataka, India
}

\author{
Prabhudev Jagadeesh M.P \\ Professor \\ Department of CSE \\ JSSATE, Bangalore, Karnataka, India
}

\begin{abstract}
Mobile Ad hoc network (Manet) is a collection of movable wireless mobile hosts. Manet does not have any fixed infrastructure. The real time Applications like video conferencing and web casting require quality of service $(Q o S)$ support. In recent years most of the QoS routing protocol have been implemented to provide bandwidth aware QoS routing. But these protocols cannot estimate bandwidth accurately due to fading and shadowing effects and variation in the existing traffic generated by neighbor nodes. Therefore this issue is open in the research field of Manet. IEEE 802.11 does not perform well in terms of throughput and delay when the traffic load increases. Admission control is needed in order to support real time application. Supporting QoS in IEEE 802.11 based ad hoc network needs available bandwidth for a route from source to destination. To reserve bandwidth for QoS routing, local bandwidth at each node should be determined. In this paper a new BWA (bandwidth aware) technique is proposed and incorporated in AODV. The BWA-AODV protocol provides better QoS routing in terms of bandwidth, packet delivery ratio end to end delay, and energy consumption compared to AODV and DSR protocol.
\end{abstract}

Keywords- QoS Routing, Bandwidth Estimation, Admission control, Manet.

\section{INTRODUCTION}

Each day, users need more quality of service for their applications. Manet is used in many applications due to infrastructure less support [1]. Manet mostly uses IEEE 802.11 protocol in distributed coordination mode. IEEE 802.11 DCF medium access scheme does not need centralized coordinator. Hosts can join and leave the network. Bandwidth is an important parameter for QoS in Manet because the network topology is highly dynamic. The bandwidth available between two adjacent hosts can be defined as the available data rate that can be sent between these two adjacent hosts without affecting the performance of existing flow in the network. In fact available bandwidth is used for optimal path selection in Manet [2]. Our goal is to estimate the available bandwidth to ensure QoS routing of new flow and ensure that existing flow should not be affected. To measure the amount of remaining bandwidth, each node uses local perception of time period the medium is idle. This information can provide an indication of the remaining available bandwidth. Our MAC uses RTS-CTS CSMA scheme.

\section{EXISTING APPROACHES}

Several bandwidth estimation techniques can be used to estimate the available bandwidth. In this section we discuss the different bandwidth estimation techniques for QoS routing in ad hoc network.

\section{A. Distributed Admission Control Protocol}

JooSang Youn et al; 2011 proposed DACP. This protocol is based on the implementation of AODV and uses RREQ for admission control. Hello message is used to count the number of contending nodes in the interference range of sender. Thus reduces the network overhead. DACP more accurately estimates the local available bandwidth by using cross layer approach between MAC and AODV routing protocol. DACP estimates the bandwidth available at each node for end-to-end provision. Bandwidth estimation at MAC layer and admission control for admitting new flow is done based on per hop basis [3]. Thus DACP minimizes the complexity of creating QoS session. Result shows that DACP provides guaranteed end-toend bandwidth and minimizes control overhead, compared to existing admission control mechanism.

\section{B. Core Extraction Distributed Ad hoc Routing (CEDAR)}

P Sinha et al; 2002 proposed CEDAR. It is a QoS enabled protocol. It dynamically forms the set of core in the network. All the nodes are members of the core or one hop away of the core node [4]. Available bandwidth information of every core's is distributed to all other cores. The routing overhead is reduced because only core node needs to keep information of bandwidth. Route is created only when it is needed and it is 


\section{International Journal of Engineering Applied Sciences and Technology, 2019 Vol. 4, Issue 6, ISSN No. 2455-2143, Pages 66-71 \\ Published Online October 2019 in IJEAST (http://www.ijeast.com)}

done by the core nodes. Core Extraction: The job of core is to minimize the number of hosts that are engaged in the interchange of network topology and information of available bandwidth. The aim of forming the core is to set up a core so that each host will be a core host or a neighbor host of the core host. Each core host keeps information of local topology and also perform discovery of route and call admission control. "Link state propagation": In CEDAR QoS routing can be provided by propagating the available bandwidth information among the core hosts [5]. When the available bandwidth of "core node" changes, information of small change in bandwidth is stored temporally and only information of stable bandwidth is distributed among the core hosts. Route Computation: it creates a core route between source and destination through the core nodes and uses the local information provided by the core. CEDAR performs "call admission" and dynamic rerouting of ongoing paths.

\section{Ad hoc QoS On-Demand Routing (AQOR)}

Qi Xue el al; 2003 proposed AQOR. This is a QoS enabled reactive routing protocol. Source node floods RREQ. The RREQ packet contains the bandwidth and delay requirements. Intermediate host performs admission control according to their available bandwidth. The intermediate host in the route table creates an entry with a time out period, if it has sufficient bandwidth to support request. If initiator node does not receive reply within the provided time, the entry will be erased [6]. Thus reduces overhead. Each host includes its reserved bandwidth in the beacon message for admission control. The traffic that affects the node is the sum of node's neighbor traffic. The end-to-end delay and available bandwidth are used to select the QoS route with sufficient bandwidth [7]. When route breaks, reserved resources are released at each node using temporary reservation. Node invalidates the reservation if it does not receive data for a short period of time. The adaptive route recovery incorporates link break detection and triggering of route repair at the destination, when there is a violation of QoS and time out for resource reservation at the destination occurs.

\section{Ticket-Based QoS Routing}

S Chen et al; 2002 proposed Ticket based QoS routing. During route discovery process tickets are distributed to estimate bandwidth or delay and minimize the flooding. Yellow ticket is used to find optimal route. Green ticket is used to determine low cost route. To find a route with minimum delay intermediate node forwards numbers of yellow tickets to their neighbor nodes [8]. If delay exceeds in the intermediate nodes than intermediate node marks the ticket as invalid. The low cost path with valid ticket is chosen by the destination node. To estimate current available bandwidth, this technique uses a weight function which includes old and new bandwidth or delay information.

\section{E. Triggered Based Distributed QoS Routing}

Swades De et al; 2002 proposed TDR. It is region based routing protocol. This protocol uses a "local database, an activity database", soft reservation, admission control for route discovery and prediction of route break for QoS support. Each host keeps an activity database and a local neighbor database. Host sends periodically beacon message that carries its region and mobility information. The neighbor node that receives this beacon keeps the power level of this beacon, information of mobility and location in its local neighbor database. Each node that is involved in the transmission of data keeps an activity database [9]. This database contains source ID, session ID, source location, destination ID, maximum bandwidth required, maximum allowed delay, next node ID, destination location, and distance from source. When source host wants to discover a route, it floods route request message to its neighbor hosts for stable route, the neighbor node that receives power level higher than predefined threshold is considered as optimal links for the route. If source cache has the destination location than selective route request is used [10]. The intermediate nodes while forwarding route request message compare their available bandwidth with requested bandwidth and check that whether it can accept the request. The destination node sends ACK when it receives the first route request. After receiving this ACK all intermediate nodes update their reserved bandwidth in the database. To detect route breaks the three types of power levels are used: $T_{h r 1}>T_{h r 2}>P_{c r}$. Where $P_{c r}$ is the critical limit. If the received power is between $T_{h r 1}$ and $P_{c r}$ than intermediate host sends a request for rerouting to the source host. After receiving the request host starts rerouting process. The intermediate node starts rerouting process when power level is between $T_{h r 1}$ and $T_{h r 2}$.

\section{F. Service Differentiation in Wireless Ad hoc Network (SWAN)}

Gahng Seop Ahn et al; 2002 proposed SWAN. It is a stateless and distributed network model. It uses feedback mechanism to support real time application. It uses rate controller for UDP and best effort traffic to support bandwidth and delay of real time application [11]. Rate controller aborts best effort traffic to support bandwidth requirement of real time traffic. Swan uses three main elements: "packet classifier, admission controller and rate controller" as shown in fig 1. Classifier is used to differentiate between real time network flow and best effort network flow. Shaper is used to process best effort traffic. The shaper's aim is to delay best effort data packet based on the rate estimated by the rate controller. The acceptance of new real time traffic is done at the source host. Thus in between hosts do not perform admission control [12]. Source node uses probing technique to measure the end-to-end available bandwidth. In case of inaccurate admission control or traffic, rerouting is done for real time sessions. Swan uses explicit congestion control (ECN) to dynamically control UDP real time flow. The demerit of SWAN is that it can only 


\section{International Journal of Engineering Applied Sciences and Technology, 2019 \\ Vol. 4, Issue 6, ISSN No. 2455-2143, Pages 66-71 \\ Published Online October 2019 in IJEAST (http://www.ijeast.com)}

support weak service guarantee [13], [18]. SWAN is assumed to be stateless but intermediate nodes may need to keep information of new flow and old flow that passes through them.

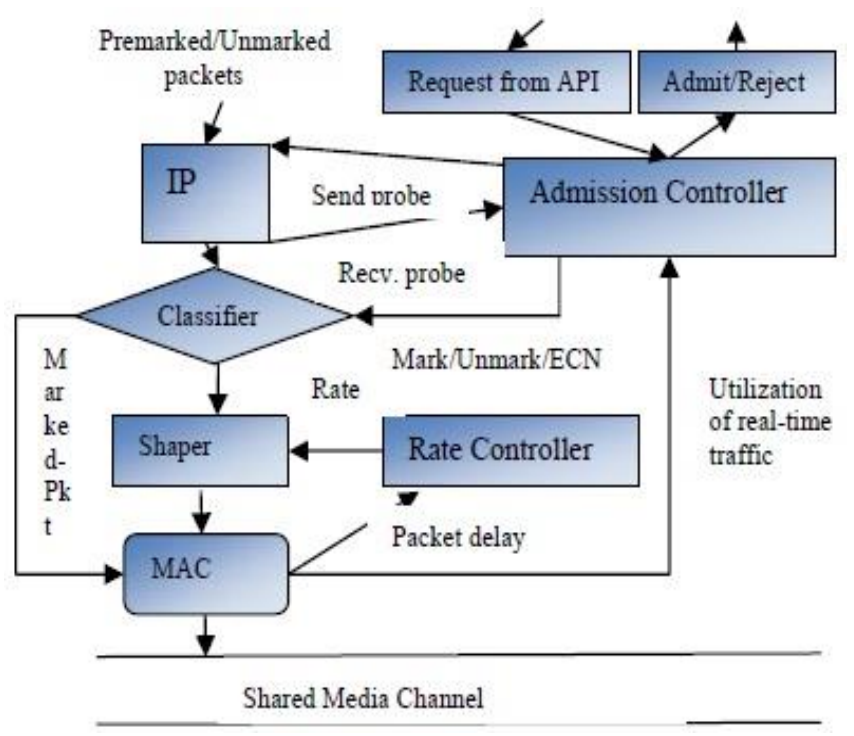

Fig. 1. Components of SWAN model.

Since source host performs admission control and use probing packet, it may result in heavy traffic.

\section{G. OLSR-Based QoS Routing}

Ying Ge et al; 2003 proposed this protocol. In this QoS schemes are integrated into the "optimized link state routing (OLSR)" protocol to choose a route with more bandwidth. This technique does not change the routing process of OLSR, but it selects different metrics to set the "multipoint relay (MPR)" set so that it can find a path with highest bandwidth. OLSR is an improved version of the link state flooding algorithm. An MPR is formed by choosing a set of nodes. Broadcast packets are sent only to the nodes that belong to MPR set [15], [19]. Therefore overhead is minimized partially compared to basic flooding where each node has to forward broadcast packets. In OLSR one hop neighbors that cover twohop neighbors are selected for MRP set for minimizing the number of MPR sets. But in MPR set nodes with low available bandwidth may be chosen that enable the link to go via nodes with low available bandwidth. Therefore criteria for selecting MPR set are modified.

Three schemes are used:

- The one hop neighbor which has largest bandwidth to reach the same number of two-hop neighbors, the current node with highest bandwidth is chosen for MPR set.

- Choose one-hop neighbors with largest bandwidth as the MPR set.

- Choose the MPR set that satisfies the criteria that twohop neighbors have sufficient bandwidth.
Result shows that the schemes a and c work well. Thus in QoS based OLSR, when the network is deployed, the "MPR" set is chosen based on the available bandwidth of the nodes. RREQ is broadcasted in MPR set. MPR set satisfies that the path with highest bandwidth will be chosen. Advantage of this method is that it is bandwidth estimation enabled algorithm. It uses carrier sense mechanism to estimate the available bandwidth. But this protocol is suitable for static topology [20]. This protocol does not consider the effect of mobility and change in bandwidth. Thus maintenance of route is not necessary.

\section{Available BandwidTh Estimation}

Several techniques are used to estimate the available bandwidth. The simple way is to measure the channel utilization and subtract it from the channel capacity [16]. The different techniques are suggested such as queue length, channel busy time and congestion window. The probability of collisions, Queue length and congestion window provide very less information when node does not transmit data. The channel busy time is a passive technique to measure channel usage. In wireless ad hoc network, carrier sensing allow hosts to identify transmitting, busy and receive state [17], [19]. We define the busy period as the total time interval in which host transmits, receives packet and senses transmission.

\section{A. Comparative Evaluation}

The objective of this section is to evaluate and compare the performance of best effort AODV protocol with proposed BWA-AODV (Bandwidth Aware-AODV). If a node has a data frame to send, it uses carrier sensing to identify the current channel state. If channel is busy, the node stops transmission of packets and wait for back-off time.

$\mathrm{T}_{\text {Backoff }}=R \times$ aslotTime

Where $\mathrm{T}_{\text {Backoff }}$ is the backoff period, $\mathrm{R}$ is a random integer distributed within the range $[0, C W]$, aslotTime is a slot duration. In this method back-off duration is predicted based on various records. If $T_{O}$ is the observation period, $T_{b}$ is the duration of back-off in second then $\mathrm{T}_{\text {Backoff }}$ in the observation period can be estimated by (1).

$$
\mathrm{T}_{\text {Backoff }}=T_{O} \times \frac{1}{n} \times \sum_{i=1}^{n} T_{O}
$$

The node records back-off information and back-off duration within a given period of time. When a node needs to estimates bandwidth, it takes NAV information from its neighbor and estimate the total busy time period of channel within the observation period. The available bandwidth at a node can be estimated by (2).

$$
\mathrm{BW}=\frac{\mathrm{T}_{\mathrm{O}}-\sum_{\mathrm{i}=1}^{\mathrm{n}}\left(\mathrm{NAV}_{\mathrm{i}}+\mathrm{T}_{\mathrm{DIFS}}+\mathrm{T}_{\mathrm{RTS}}-\mathrm{T}_{\text {Backoff }}\right)}{\mathrm{T}_{\mathrm{O}}} \times \mathrm{C}
$$


This bandwidth estimation technique is incorporated in AODV, when a host receives a route request it performs admission control, if available bandwidth is greater than or equal to requested bandwidth, node accept request otherwise rejects request.

IV. Simulation Results

Table-1 Simulation parameters.

\begin{tabular}{|c|c|}
\hline Parameters & Value \\
\hline Number of nodes & 30 \\
\hline Simulation time & 80 Seconds \\
\hline Simulation Area & $500 \times 500 \mathrm{~m}^{2}$ \\
\hline Traffic source & CBR \\
\hline Agent & UDP \\
\hline Routing Protocol & AODV, DSR BWA-AODV \\
\hline Packet Size & 512 Bytes \\
\hline MAC & IEE $802 \_11$ \\
\hline Propagation Model & Two Ray Ground \\
\hline Basic rate & $0.5 \mathrm{Mbps}$ \\
\hline Data rate & $2 \mathrm{Mbps}$ \\
\hline
\end{tabular}

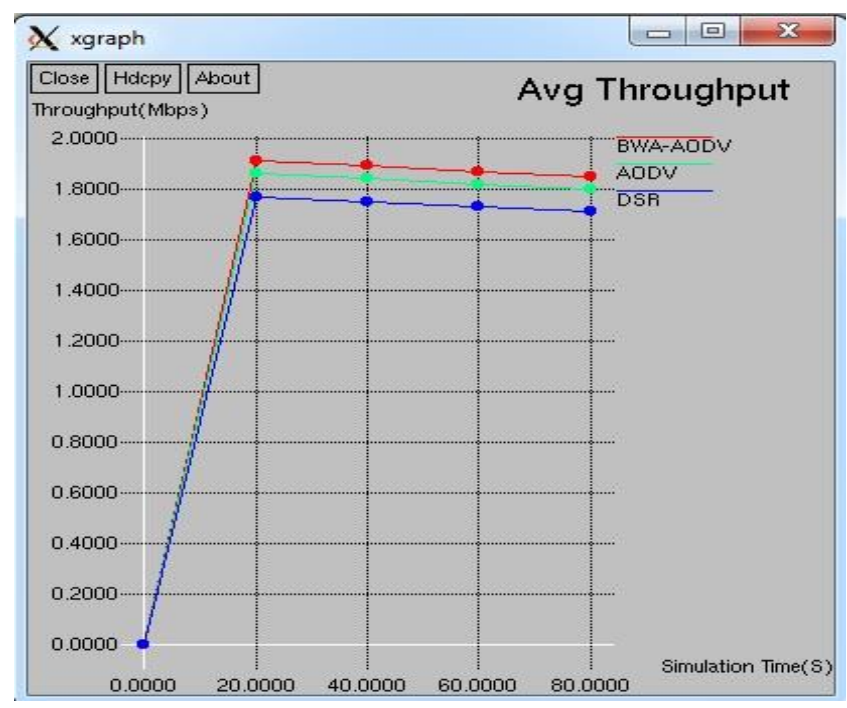

Fig. 2. Average throughput.

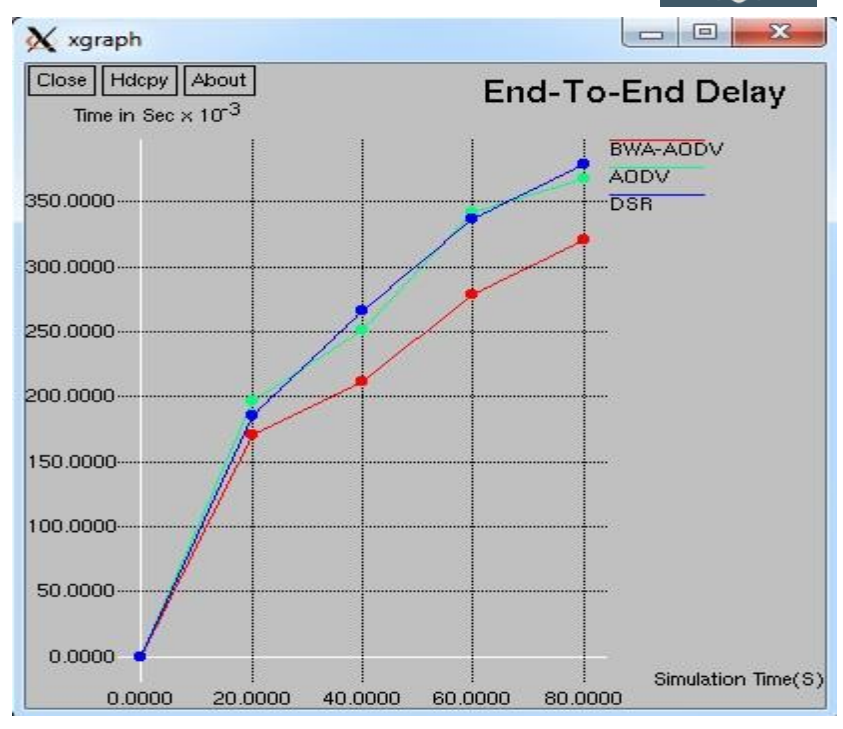

Fig. 3. Average end to end delay.

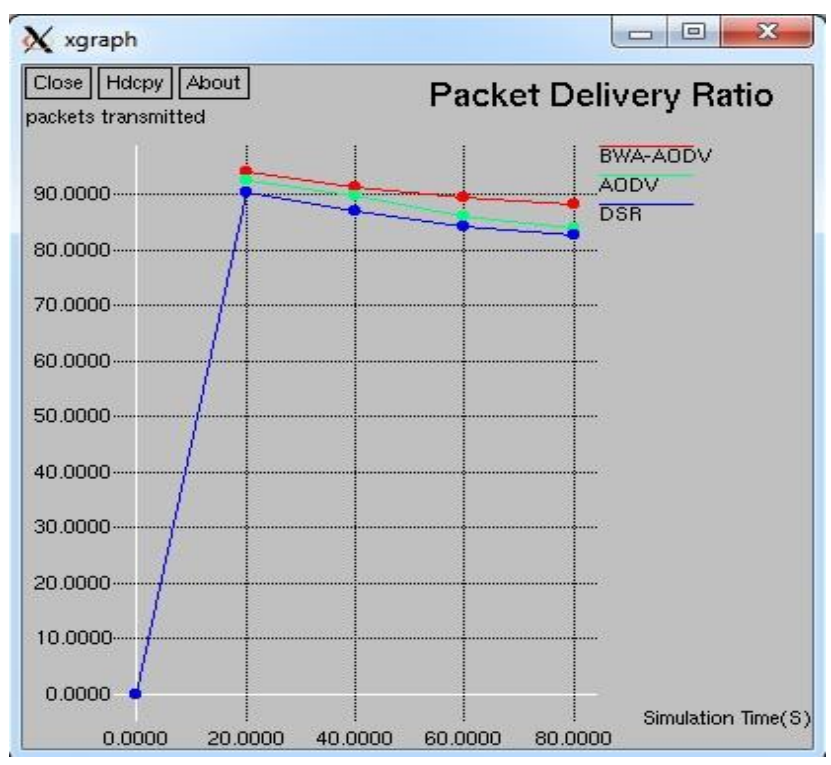

Fig. 4. Packet delivery ratio. 




Fig. 5. Average energy consumption.

Fig. 2 shows average throughput using three different protocols, one is QoS enabled routing protocol called BWAAODV and two best effort routing protocols AODV and DSR. First we observe that BWA-AODV has achieved a throughput of $1.9 \mathrm{Mbps}$. AODV has achieved a throughput of $1.8 \mathrm{Mbps}$ and DSR has achieved average throughput of $1.7 \mathrm{Mbps}$.

\section{CONCLUSION AND FUTURE WORK}

In this paper a BWA technique has been implemented and incorporated into AODV. As we can observe that the throughput using BWA-AODV is much better than best effort AODV and DSR because AODV and DSR do not include any bandwidth estimation technique and admission control scheme. BWA-AODV delay is less compared to AODV and DSR. Packet delivery ratio of BWA-AODV better compared to AODV and DSR. Energy consumption in case of BWAAODV is less compared to AODV and DSR. In future we plan to incorporate bandwidth aware routing protocol into NS 2.35. In this embedded network simulator we will be able to see the performance of routing protocols in highly network topology. The method of bandwidth estimation will be combined with admission control to support real time application and avoid rerouting of same packet. In this way we can improve QoS routing in highly dynamic network.

\section{REFERENCES}

[1] Nicolas and dopolus, (2018). Ad hoc Mobile and Wireless Network, Springer, September. (pp.5-7).
[2] R. Asokan, (2010). Areview of Quality of Service (QoS) routing protocol for mobile Ad hoc networks. in Proc. IEEE (ICWCSC2010), Chennai, India, ( pp. 1-6).

[3] R. Sivakumar, P. Sinha and V. Bharghavan, (1999). "CEDAR: a core-extraction distributed ad hoc routing algorithm” IEEE J.Select. [26] Areas Commun. Vol. 17, (pp.1454-1465).

[4] Redouane Belbachir, Mekkakia Maaza Zoulikha and Ali Kies. (2012).IEEE wireless and networking conference mobile and wireless networks. (pp. 2608-2613).

[5] H. Zhao, E. Garcia-Palacios, J. Wei, and Y Xi. (2009). Accurate Available Bandwidth Estimation in IEEE 802.11-Based Ad hoc Networks. Computer Communications. (pp. 1050-1057).

[6] L.A. Latif, A. Alliand, C.C. O. (2005). Location based geo-casting and forwarding (LGF) routing protocol in mobile ad hoc network", Proceedings on the Advanced Industrial Conference on Telecommunications/Service assurance with partial Intermittent Resources. (pp. 536541).

[7] L.Li; and C.Li. (2006). A hierarchical QoS multicast routing protocol for mobile ad-hoc networks", Chinese Journal of Electronics 15(4), ( pp. 573- 577).

[8] C.R; Lin and J.S. Liu..(1999). QoS routing in ad hoc wireless networks", IEEE J. Select. Areas Commun, Vol. 17, (pp. 1426-11438).

[9] J. Hong. (2004). Efficient on-demand routing for mobile ad hoc wireless access networks", IEEE journal on selected Areas in Communications 22. ( pp. 11-35).

[10] G. Bianchi. (2000). Performance analysis of the IEEE 802.11 distributed coordination function", IEEE Journal on Selected Areas in Communications 18 (3). (pp.535547).

[11] Zhenhui Yuan. (2012). MBE: Model-Based Available Bandwidth Estimation for IEEE 802.11 Data Communications. IEEE Transactions on Vehicular Technology, vol. 61, no. 5, (pp. 2158-2171).

[12] Calafate C. T., Manzoni P., and Malumbres M. P., (2005). Supporting Soft Real-Time Services in MANETs using Distributed Admission Control and IEEE 802.11e Technology, 10th IEEE Symposium on Computers and Communications, (pp. 217-222).

[13] Liebeherr J., Fidler M., and Valaee S. (2010). A SystemTheoretic Approach to Bandwidth Estimation", IEEE/ACM Transactions on Networking”, vol. 18, no. 4, (pp. 1040-1053).

[14] David Espes; and Zoubir Mammeri. (2009). QoS-aware Routing for Real-Time and Multimedia Applications in Mobile Ad Hoc Networks. International Conference on Real-Time and Network Systems. ( PP.67-74).

[15] Xiongwei Ren and Hongyuan Wang. (2005). A Soft Bandwidth Constrained QoS Routing Protocol for Ad 
Hoc Networks", International Conference on Mobile AdHoc and Sensor Networks. (pp 548-559).

[16] Rajeev Kumar. (2017). A comprehensive analysis of MAC protocols for Manet,. IEEE International Conference on Electrical, Electronics, Communication, Computer and Optimization Techniques (ICEE CCOT), (pp.56-58).

[17] Rajeev Kumar and Ranjeet Kumar. (2016). Reactive Unicast and Multicast Routing Protocols for Manet and Issues A Comparative Analysis" International Conference on internet of things, International Journal of Engineering and Technology (IJERT), Volume 4, Issue 29. ( pp. 135137).

[18] Xu Zhen and Y Wenzhong. (2013). Bandwidth-Aware Routing for TDMA-Based Mobile Ad hoc Networks. IEEE The International Conference on Information Networking (ICOIN). ( pp.637-642).

[19] Obara, H., Koseki, S., and Selin, P. (2012). Packet train pair: A fast and efficient technique for measuring available bandwidth in the internet", in SICE annual conference. (pp. 1833-1836).

[20] Wu Dapeng, Yan Zhen, Sun Bing, Xu Chunxiu, and Wu Muqing. (2008). Improving Accuracy of Bandwidth Estimation Based on Retransmission Predicting in MANET. 4th International Conference on Wireless Communications, Networking and Mobile Computings. (pp.1-4). 